\title{
Desmonte en Parques Eólicos en Ejido de Puerto Madryn, Chubut, Argentina.
}

\section{Vegetation clearance at wind farms at Puerto Madryn, Chubut, Argentina.}

Presentación: 27/04/2020

Aprobación: 25/06/2020

Jonatan Hojman

Cauquén SRL - Argentina

jhojman@cauquensrl.com

\section{María Pía Di Nanno}

Facultad Regional Chubut, Universidad Tecnológica Nacional - Cauquén SRL - Argentina

piadinanno@yahoo.com.ar

\section{Resumen}

El desmonte y la fragmentación del hábitat que eso produce, es uno de los impactos ambientales más relevantes en la etapa de construcción de los parques eólicos. En este trabajo se evaluó el desmonte asociado a los tres parques eólicos ubicados dentro del ejido de la ciudad de Puerto Madryn, Chubut, Argentina. El desmonte se determinó a partir de técnicas de teledetección con procesamiento de imágenes satelitales. Se comparó el desmonte parque por parque y valor promedio contra estándares de la industria y se determinó el desmonte asociado a proyectos eólicos de la urbanización. Los resultados demostraron que el desmonte de los parques corresponde a unas 996 has, representando un 0,81\% de la superficie del ejido en la actualidad. Asimismo el área de impacto total directo promedio en has/MW fue de 1,70 , valor que se encuentra dentro de los rangos de la industria.

Palabras clave: parque eólico; desmonte; Puerto Madryn.

\begin{abstract}
Vegetation clearance is one of the most relevant environmental impacts at the construction stage of the wind farms. This report evaluated the vegetation clearance of the three wind farms located within Puerto Madryn city area. Vegetation clearance was assessed through the use of remote sensing techniques with satellite image processing. Vegetation clearance was compared against industry standards wind farm by wind farm and on an average basis and also against the total vegetation clearance caused by the city urbanization process. Results show that vegetation clearance associated to the wind farms amounts to 996 hectares representing $0,81 \%$ of the city area. Also the average total direct impact area was 1,70 hectares per MW, which is within industry standards.
\end{abstract}

Keywords: wind farm, vegetation clearance; Puerto Madryn. 


\section{Introducción}

El espacio o superficie ocupada por los parques eólicos puede ser medido de distintas formas. El área desmontada o que presenta remoción de la vegetación incluye el espacio ocupado por las plataformas de las turbinas, caminos de acceso, estacionamientos, oficinas, red colectora, y otros trabajos civiles. Esta área es variable, pero según Ledec et al. (2011), ronda entre 1 y 2 hectáreas por MW. Asimismo, parte de este impacto a nivel de desmonte es permanente, como en el caso de caminos y partes de la plataforma, y otro es temporal, como en el caso de canteras -de existir-, obradores, red colectora y otros (ver Figura 1). Por otro lado, el área ocupada por el parque comprende todo el sector de aerogeneradores, la estación elevadora y las demás instalaciones necesarias. Estos sectores son normalmente delimitados por alambrados (de 3 o 5 hilos). Normalmente existe también un área buffer rodeando los parques donde se prohíbe la plantación de árboles o la instalación de estructuras altas para no afectar el flujo de viento a las turbinas. Esta área varía considerablemente según las condiciones del viento, topografía y otros factores, pero suele rondar entre $10 \mathrm{y}$ 30 ha/MW (Ledec et al., 2011). Por otro lado si consideramos el impacto visual de las instalaciones, tomando en cuenta el área desde la cual el parque es visible, este área es variable según topografía y altura de los aerogeneradores y puede involucrar hasta un radio de $30 \mathrm{~km}$ (Ledec et al., 2011).

En el presente trabajo se adopta la definición de Denholm et al. (2009) en lo referido al área de impacto directo total (área ocupada por plataformas, caminos, subestación, edificios o cualquier otra infraestructura que ocupe el suelo o que cree superficies impermeables). Esta diferenciación en relación con el área total de proyecto se realiza teniendo en cuenta que no se interfiere con otros usos como la agricultura, cría de ganado o provisión de servicios ecosistémicos, correspondiendo normalmente el $90 \%$ de la superficie a área disponible para otros usos como los indicados (Fritsche et al., 2017).

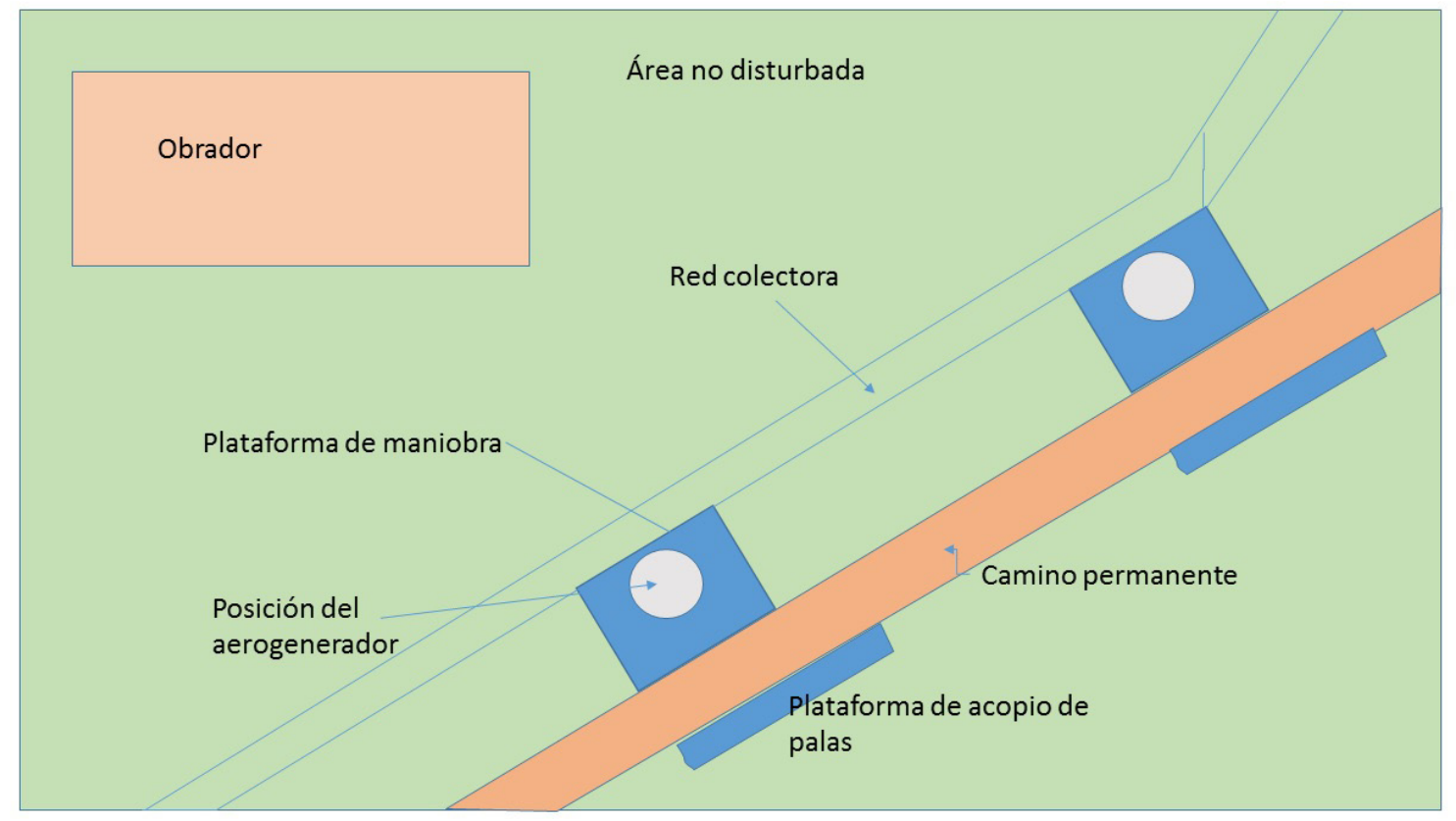

Figura 1. Componentes de impacto directo en un parque eólico (permanente y no permanente). 
Esta área de impacto directo se divide a su vez en área de impacto directo no permanente (obrador, caminos temporales, sectores de acopio, canteras, etc.) e impacto directo permanente (edificios, caminos, parte de la plataforma). Las áreas de impacto no permanente son normalmente sometidas a trabajos de remediación, que en la zona de interés del presente trabajo normalmente consisten en la promoción de la revegetación natural (típicamente gestión diferencial del topsoil al desmontar; posterior colocación de topsoil y escarificado de las áreas intervenidas).

La fragmentación del hábitat producida por el desmonte y las superficies afectadas de suelos son uno de los impactos ambientales más relevantes en la etapa de construcción de los parques eólicos, y se adoptan medidas de control para minimizarlo y gestionar correctamente el topsoil a los efectos de remediar las áreas intervenidas correctamente.

Nuestro objetivo es verificar cual es el área de impacto directo afectada por los parques eólicos incluidos dentro del ejido de la ciudad de Puerto Madryn, Chubut, Argentina. Por otro lado, comparar dicha superficie contra los estándares normales de la industria.

El ejido de esta ciudad se amplió el pasado 19 de diciembre de 2019, mediante Ley Provincial XVI N 101 y Decreto $N^{\circ} 1609 / 19$ (Boletín Oficial Chubut $N^{\circ} 13314$ ), incorporando dentro del mismo a tres parques eólicos, detallados a continuación:

-Parque eólico Madryn (etapas 1 y 2) de Genneia (PEMAD de ahora en adelante);

-Parque eólico Chubut Norte (etapas 1, 2, 3 y 4) de Genneia (aún en construcción las últimas 3 etapas; PECHN ahora en adelante)

-Parque eólico El Llano de GENPAT (etapas 1, 2 y 3; PEEL de ahora en adelante).

\section{Desarrollo}

En todas las etapas de este documento, se utilizó el software que se describe a continuación para la elaboración del presente informe a partir de técnicas de teledetección. El software utilizado para el procesamiento de los datos de las imágenes satelitales fue el QGIS (versión 3.4.2. with GRASS 7.4.2). Las imágenes satelitales utilizadas corresponden al satélite Sentinel 2, de la misión Copernicus de la agencia espacial europea (ESA, según sus siglas en inglés), de la Comisión Europea, descargada a través del sistema Copernicus Hub ${ }^{1}$ y EO Browser ${ }^{2}$.

El conjunto de datos crudos corresponde al Sentinel-2-SA-L1C, fecha: 22/01/2020, y fueron procesadas por el complemento QGIS Semi Automatic Classification Plugin ${ }^{3}$.

Las imágenes fueron clasificadas por el complemento QGIS Semi Automatic Classification Plugin con 4 macroclases (Desmonte, Vegetación, Agua, Urbanidad) y entre ellos 15 clases totales. Para la evaluación, y en función de las bandas que contiene el Sentinel 2, se utilizaron las RGB y el infrarrojo cercano (2,3,4 y 8), sumado a un NDVI (Índice de Vegetación Diferencial Normalizado, por sus siglas en inglés) generado por el software mencionado. La resolución corresponde a $25 \mathrm{~m} 2$ (pixel de $5 \mathrm{~m} \mathrm{x} 5 \mathrm{~m})^{4}$. Con la clasificación realizada se generó un archivo vectorial del que automáticamente, por medio del software QGIS, se obtuvo el área de la superficie afectada.

La información referida a los proyectos fue obtenida de los estudios de impacto ambiental y sus correspondientes declaratorias de impacto ambiental, que son información de acceso público atento a que se publican en la web del Ministerio de Ambiente y Control del Desarrollo Sustentable, autoridad de control ambiental a nivel provincial. Lo mismo se hizo para las canteras de áridos asociadas a los proyectos que transitan un procedimiento de EIA 1 https://scihub.copernicus.eu/ 
independiente, regido por la Ley Nacional $\mathrm{N}^{\circ} 24.585$.

\section{Resultados y discusión}

Se evaluaron los resultados de los tres citados parques eólicos, que representan un total de 156 aerogeneradores y $587 \mathrm{MW}$ totales instalados/en proceso de instalación. Los datos de superficie de los parques eólicos, cantidad de aerogeneradores y potencia instalada o a instalar fueron obtenidos a través de la revisión de los estudios de impacto ambiental de los parques eólicos (Genneia, 2015; Genneia, 2019; Emgasud y Emgasud Renovables, 2009; GENPAT y Aluar, 2017; Genneia, 2015; Genneia y Emgasud, 2012; Genneia, 2014).

Se presenta a continuación la Figura 2, donde se observa el ejido de Pto. Madryn y el desmonte asociado a los parques eólicos.

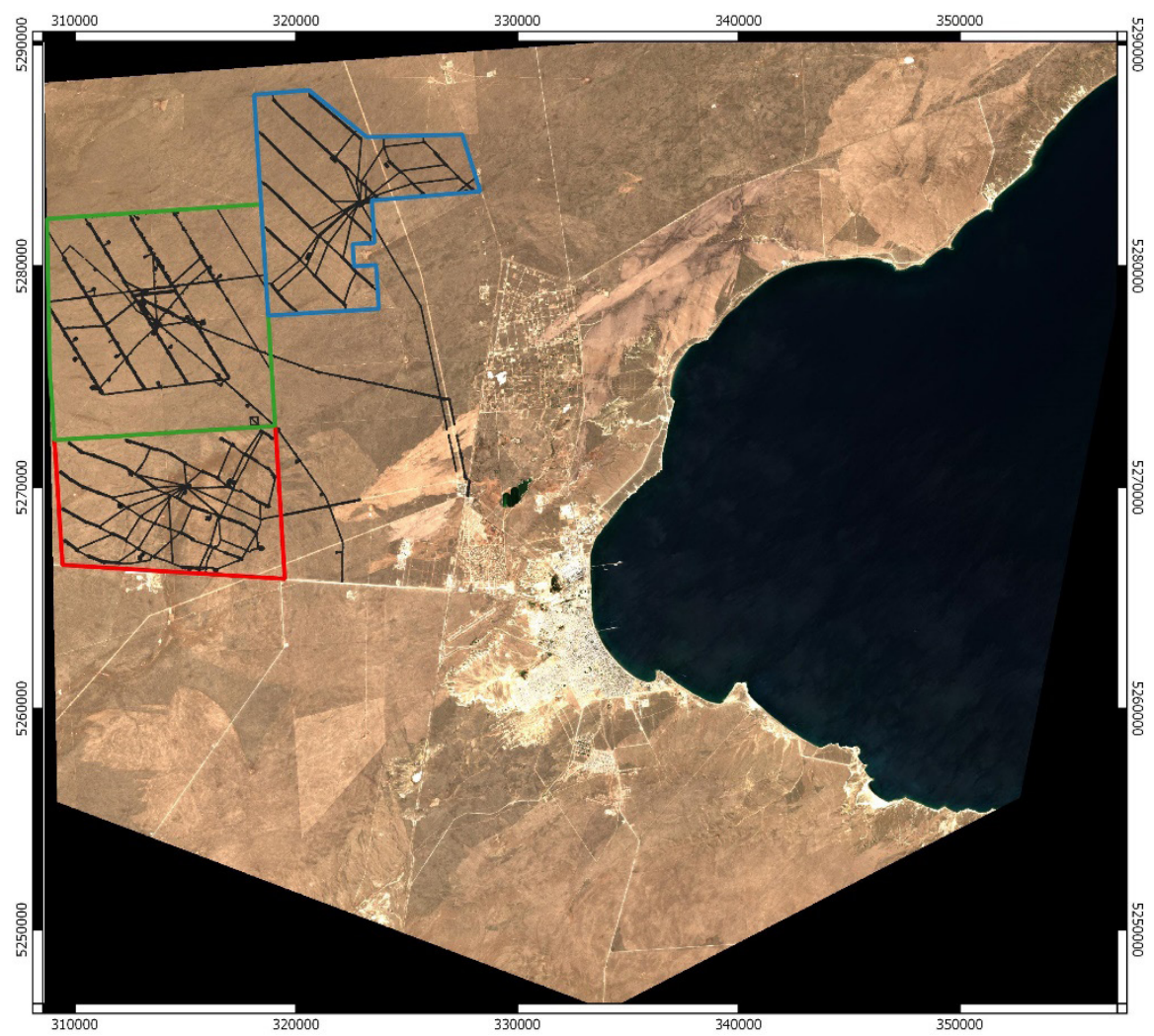

Figura 2. Análisis de desmontes con sensores remotos de parques eólicos en ejido de Puerto Madryn, Chubut, Argentina.
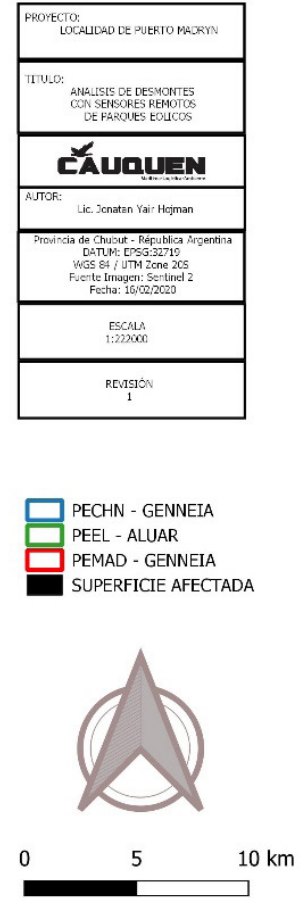

A continuación en la Tabla 1 se analizan los resultados obtenidos: 


\begin{tabular}{|l|c|c|c|c|}
\hline $\begin{array}{l}\text { Indicador } \\
\begin{array}{l}\text { Área de impacto } \\
\text { directo total (has) }\end{array}\end{array}$ & PECHN & PEEL & PEMAD & $\begin{array}{c}\text { Total PARQUES EOLICOS } \\
\text { EN EJIDO MADRYN }\end{array}$ \\
\hline $\begin{array}{l}\text { Superficie total del } \\
\text { parque eólico (has) }\end{array}$ & $6.283,4$ & 927,1 & 298,5 & 996 \\
\hline $\begin{array}{l}\text { Porcentaje de } \\
\text { afectación por } \\
\text { parque }\end{array}$ & $4,30 \%$ & $4,31 \%$ & $5,06 \%$ & 22.107 \\
\hline $\begin{array}{l}\text { Cantidad de } \\
\text { aerogeneradores } \\
\text { instalados/ } \\
\text { en proceso de } \\
\text { instalación al 16 de } \\
\text { Febrero de 2020. }\end{array}$ & 46 & 48 & $5.903,1$ & 156 \\
\hline $\begin{array}{l}\text { Potencia } \\
\text { instalada/a instalar } \\
\text { (MW) }\end{array}$ & 199,8 & 164,8 & 222,3 & 587 \\
\hline $\begin{array}{l}\text { Área de impacto } \\
\text { directo total por MW } \\
\text { (has/MW) }\end{array}$ & 1,35 & 2,59 & 1,34 & 1,70 \\
\hline
\end{tabular}

Tabla 1. Resultados del análisis de desmontes con sensores remotos.

Téngase en cuenta que en el caso de PECHN, se encuentra en etapa de montaje para las últimas etapas del parque, pero los desmontes correspondientes a plataformas y otra infraestructura ya han sido ejecutados.

Es importante aclarar que hay un pequeño sector afectado por incendios donde el modelo no fue capaz de diferenciar desmonte de la picada de la línea de $132 \mathrm{kV}$ de PEMAD respecto del incendio, con lo cual se está subestimando ese sector. Esto ocurrió también en un sector mucho menor de la LAT de $132 \mathrm{kV}$ de PEEL y PECHN, al norte de la acometida a la ET de TRANSENER. Sin embargo se consideran despreciables respecto del total.

Cabe destacar asimismo que parte de estas áreas no son de afectación permanente y fueron o serán sometidas a procedimientos de promoción de la revegetación natural, con lo que la superficie afectada deberá ir disminuyendo con el tiempo.

De acuerdo a la imagen, la superficie del ejido de Puerto Madryn es en la actualidad de unas 123.620 has y el desmonte asociado a la urbanización corresponde a 4.678 hectáreas (3,78\% del ejido). El desmonte asociado a los parques eólicos respecto del ejido de dicha localidad corresponde al $0,81 \%$. Por otro lado, respecto del desmonte total de la ciudad, el asociado a los parques eólicos corresponde al $21 \%$. Cabe destacar que este desmonte se llevó a cabo en su totalidad a partir de 2017 cuando dichas obras iniciaron, con lo cual es un incremento relevante en el último tiempo.

Se observa por otro lado que más del $95 \%$ de la superficie en los parques eólicos, está disponible para otros usos. Esto se condice con el porcentaje referido por Fritsche et al., 2017. Si bien estos campos estaban dedicados a la actividad ganadera (ganado ovino), en la actualidad la mayoría de los mismos no estaban en producción. Eventualmente la nueva infraestructura puede promover otro tipo de producciones alternativas. 


\section{Benchmarking}

Se presenta la Tabla 2 donde se detalla la comparativa del desmonte relevado en Puerto Madryn respecto de los estándares de la industria.

Con los datos de las imágenes satelitales exclusivamente no fue posible diferenciar el desmonte permanente del no permanente, ya que se requiere conocer en detalle el parque y las decisiones de la administración del mismo respecto de ciertas áreas como sectores de plataformas de acopio de palas y/o armado de grúas en particular, que no siempre se remedian. Sin embargo se dejan las filas para observar que, según estándares de la industria y sin contabilizar desmontes asociados a explotaciones de áridos, normalmente el área a remediar podría alcanzar hasta el 70\% del área intervenida. Esto es relevante en relación a la permanencia de los impactos asociados al desmonte, que no serán tales dentro de unos años cuando la estepa arbustiva predominante en la zona avance sobre los sectores remediados.

\begin{tabular}{|c|c|c|c|c|c|c|c|c|}
\hline Indicador & PECHN & PEEL & PEMAD & $\begin{array}{l}\text { Promedio de } \\
\text { requerimiento de } \\
\text { área }\end{array}$ & $\begin{array}{l}\text { Ledec } \\
\text { et al. } \\
(2011)\end{array}$ & $\begin{array}{l}\text { Denholm } \\
\text { et al. } \\
(2009)\end{array}$ & $\begin{array}{l}\text { Wilburn, } \\
\text { D. (2011) }\end{array}$ & $\begin{array}{c}\text { Kumar } \\
\text { et al. } \\
\text { (2017) }\end{array}$ \\
\hline $\begin{array}{l}\text { Área de } \\
\text { impacto } \\
\text { directo total } \\
\text { (has) /MW } \\
\text { instalado }\end{array}$ & 1,35 & 2,59 & 1,34 & 1,70 & $\begin{array}{c}\text { Entre } \\
1 \text { y } 2\end{array}$ & $1,0 \pm 0,7$ & -- & $1,25-1,5$ \\
\hline $\begin{array}{l}\text { Área de } \\
\text { impacto } \\
\text { directo } \\
\text { permanente/ } \\
\text { MW instalado }\end{array}$ & $\begin{array}{c}\text { Sin } \\
\text { datos }\end{array}$ & $\begin{array}{c}\text { Sin } \\
\text { datos }\end{array}$ & $\begin{array}{c}\text { Sin } \\
\text { datos }\end{array}$ & -- & -- & $0,3 \pm 0,3$ & $0,3 \pm 0,3$ & -- \\
\hline $\begin{array}{l}\text { Área de } \\
\text { impacto } \\
\text { directo no } \\
\text { permanente/ } \\
\text { MW instalado }\end{array}$ & $\begin{array}{c}\text { Sin } \\
\text { datos }\end{array}$ & $\begin{array}{c}\text { Sin } \\
\text { datos }\end{array}$ & $\begin{array}{c}\text { Sin } \\
\text { datos }\end{array}$ & -- & -- & $0,7 \pm 0,6$ & $0,7 \pm 0,6$ & -- \\
\hline $\begin{array}{l}\text { Hectáreas de } \\
\text { proyecto/MW } \\
\text { instalado }\end{array}$ & 31,4 & 60,2 & 26,6 & 37,7 & -- & $34 \pm 22$ & 34,5 & -- \\
\hline $\begin{array}{l}\% \text { de superficie } \\
\text { ocupada } \\
\text { respecto del } \\
\text { total del área } \\
\text { de proyecto }\end{array}$ & $4,30 \%$ & $4,31 \%$ & $5,06 \%$ & $4,55 \%$ & -- & -- & $2-5 \%$ & -- \\
\hline
\end{tabular}

Tabla 2. Comparativa contra estándares de la industria.

En relación al indicador de has/MW se observa que el promedio de los 3 parques presenta un valor de 1,7 has/MW, que se encuentra dentro del rango de Ledec et al. (2011) y Denholm et al. (2009), pero por encima del rango de Kumar et al. (2017). Individualmente se puede observar que el PEEL presenta un desmonte superior en has/MW, lo cual podría deberse a la ejecución de infraestructura que posteriormente se emplee en ampliaciones posteriores (caminos, electroductos, etc.).

Si comparamos el área ocupada por el proyecto por MW instalado se observa que los 
parques están en el orden de magnitud de las referencias.

Por otro lado, comparando desmonte (en cada parque respecto de la superficie del predio del parque), los desmontes están cerca del borde superior del reportado por la industria. Aquí sin embargo es importante destacar que el modelo incluye desmonte asociado a canteras de explotación de áridos, que se encuentran dentro del parque e incrementan la superficie afectada, pero no de manera permanente. La mayoría de los indicadores relevados no contemplan estos desmontes.

\section{Conclusiones}

Se observa que si bien los desmontes asociados a los parques eólicos se encuentran dentro del rango general de la industria, el impacto asociado a tal circunstancia es relevante y debe minimizarse. Para mejorar el análisis deben diferenciarse los desmontes permanentes de aquellos no permanentes e indicar asimismo si se contemplan canteras de áridos o no, para mejorar las comparaciones contra los indicadores de la industria a nivel mundial.

Se observa asimismo que si bien el porcentaje de desmonte asociado a los parques en ejido de Pto. Madryn es muy bajo (0,81\%), el mismo corresponde a más de un $20 \%$ del desmonte total asociado al ejido y ocurrió en un período acotado de tiempo.

Se plantea como trabajo a futuro relevar otros parques eólicos en Chubut a los efectos de ampliar la muestra y obtener mejores indicadores de desmonte para estimar los impactos de la actividad en la provincia. Se espera que este trabajo permita mejorar las estimaciones de área a afectar en las evaluaciones de impacto ambiental. 


\section{Referencias}

Ledec, G; Rapp, K; Aiello, R (2011). "Greening the wind. Environmental and social considerations for wind power development”. The World Bank. Disponible en: <http://documents.worldbank.org/curated/en/239851468089382658/ pdf/662330PUB0EPI00e0wind09780821389263.pdf $>$.

Denholm, P; Hand, M; Jackson, M; Ong, S. (2009) "Land-Use Requirements of Modern Wind Power Plants in the United States" National Renewable Energy Laboratory. United States Department of Energy. Office of Energy Efficiency and Renewable Energy. Disponible en: <https://www.nrel.gov/docs/fy09osti/45834.pdf>.

Fritsche, U.; Berndes, G; Cowie, A; Dale, V; Kline, K; Johnson, F; Langeveld, H; Sharma, N; Watson, H; Woods, J. (2017) "Energy and Land use". International Renewable Energy Agency (IRENA). Disponible en: <http://iinas.org/tl_files/iinas/downloads/land/ IINAS_2017_UNCCD-IRENA_Energy-Land_paper.pdf $>$.

Wilburn, D. 2011. "Wind Energy in the United States and Materials Required for the LandBased Wind Turbine Industry From 2010 Through 2030”. U.S. Department of the Interior. U.S. Geological Survey. Disponible en: < https://pubs.usgs.gov/sir/2011/5036/sir2011-5036. pdf >.

Kumar, A; Thapar, S. 2017. "Addressing land issues for utility scale renewable energy deployment in India”. TERI School of advanced studies. Disponible en: $<$ https:// shaktifoundation.in/wp-content/uploads/2018/01/Study-Report-Addressing-Land-Issuesfor-Utility-Scale-Renewable-Energy-Deployment-in-India.pdf>

Genneia. 2015. Estudio de impacto ambiental. Parque eólico Madryn Norte. Chubut. Argentina. Disponible en: <http://www.ambiente.chubut.gov.ar/2016/07/11/convocatoriaaudiencia-publica-relativo-al-proyecto-parque-eolico-madryn-norte-y-evacuacion-deenergia-presentado-por-la-empresa-genneia-s-a/>

Genneia. 2019. Estudio de impacto ambiental. Parques eólicos Chubut Norte II, III y IV. Adenda. Provincia de Chubut. Argentina.

Emgasud S.A. y Emgasud Renovables S.A.. 2009. Estudio de impacto ambiental. Parque eólico Puerto Madryn. Provincia de Chubut. Argentina.

GENPAT - ALUAR SAIC. 2017. Estudio de impacto ambiental. Parque eólico y línea de evacuación del parque eólico GENPAT. Puerto Madryn. Provincia de Chubut. Argentina. Disponible en: <http://www.ambiente.chubut.gov.ar/2017/02/20/convocatoria-a-audienciapublicas-respecto-del-estudio-de-impacto-ambiental-del-proyecto-parque-eolico-genpat-ylinea-de-linea-de-alta-tension-peglt-presentado-por-la-empresa-genp/>

GENNEIA. 2015. Estudio de impacto ambiental. Evaluación de Energía Eléctrica del Parque Eólico Madryn Norte. Provincia de Chubut. Argentina. Disponible en: <http://www. ambiente.chubut.gov.ar/2016/07/11/convocatoria-audiencia-publica-relativo-al-proyectoparque-eolico-madryn-norte-y-evacuacion-de-energia-presentado-por-la-empresagenneia-s-a/>

GENNEIA S.A. Y EMGASUD S.A. 2012. Estudio de impacto ambiental. Evacuación de Energía Eléctrica del Parque Eólico Madryn. Provincia de Chubut. Argentina. 
Genneia. 2014. Estudio de impacto ambiental. Evacuación de Energía Eléctrica del Parque Eólico Madryn. Addenda. Provincia de Chubut. Argentina.

Boletín Oficial. Provincia de Chubut. AÑO LXII - No 13314. Jueves 19 de Diciembre de 2019. Disponible en < http://boletin.chubut.gov.ar/archivos/boletines/Diciembre\%2019,\%202019. pdf $>$. 